\title{
Book Review of Rosenhouse, The Monty Hall Problem
}

\section{Leslie Burkholder}

The Monty Hall Problem, Jason Rosenhouse, New York, Oxford University Press, 2009, xii, 195 pp, US \$24.95, ISBN 978-0-19-5\#6789-8

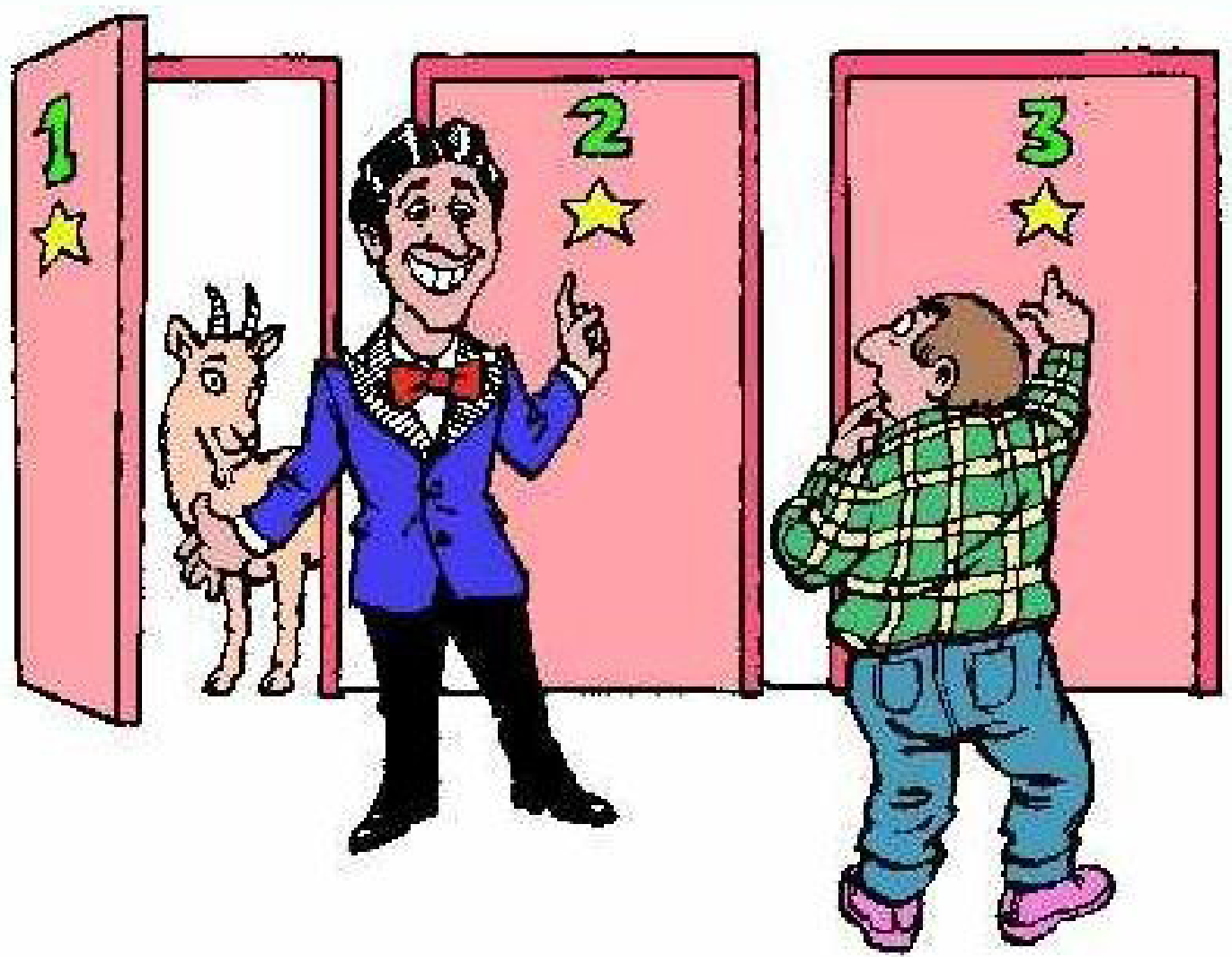

(Source www.grand-illusions.com. Used with permission.)

\footnotetext{
${ }^{1}$ University of British Columbia
} 
For the very few readers of this journal who have not heard of it here is what Jason Rosenhouse calls the "classical" statement of the Monty Hall puzzle:

You are on a game show in which you are confronted by three numbered doors. You know that one of the doors hides a car and the other two doors each hide a goat. You would like the car more than any of the goats and have no desire for one goat over the other. You cannot open the doors to see what is behind them, and you know nothing which would help you figure out where the car is. Your choice has two stages. You select one of the doors at the first stage. Suppose this is door number three. Then the host of the game show-Monty Hall-opens one of the doors and shows you what is behind it. In fact, what he opens is door number one, and what he shows you is a goat. Now comes the second stage. You can change your initial choice of door. The host has eliminated one door by opening it, but two remain. If you choose a particular door-whether by staying or switching-you get whatever is behind the door. Do you increase your chance of getting the car by changing from your initial choice of door number three to door number two?

One obvious question about this problem is: What is the correct solution and why is it the correct solution? Answers to both parts of the question are considered in this chatty and thorough survey of work on the problem in many different disciplines.

The accepted correct solution is that you increase your chance of winning the car by switching doors. In more detail, the probability that the car is behind door number two is two-thirds, and the probability or chance it is behind door number three is one-third. Since your first stage choice was door number three, you double your chances of getting the car by changing doors.

In addition to this answer, there is a rationale or explanation for these numbers that illustrates why the accepted solution is right. Using these abbreviations

car 1 = car is behind door \#1

car2 = car is behind door \#2

car3 = car is behind door \#3

showgoat 1 = host opens door \#1 and shows goat

showgoat 2 = host opens door \#2 and shows goat

showgoat3 = host opens door \#3 and shows goat

picks3 = contestant picks door \#3 at first stage

the explanation first says that the chance the car is behind door number two is provided by the conditional probability Prob(car2|[showgoat1 \& picks3]), and the chance it is behind door number three is provided by the parallel Prob(car3|[showgoat1 \& picks3]). Second, 
according to the explanation, the value of each of these conditional probabilities is determined by an instance of the Bayes theorem formula. For the first conditional probability this is:

(1)

Prob(car2 | [showgoat1 \& picks3])

$=$

$\{($ Prob([showgoat1 \& picks3]|car2) x PriorProb(car2)\}

$\{([$ Prob( [showgoat1 \& picks3] |car1) x PriorProb(car1)] + [(Prob([showgoat1 \& picks3]|car2) $x$ PriorProb(car2)] $+[($ Prob ([showgoat1 \& picks3] $\mid$ car3) $x$ PriorProb(car3)])\}

And finally, as a third part of the explanation for the numbers, certain values are taken for each of the items on the input side of the fomula. With these values plugged in the formula becomes:

(2)

$\operatorname{Prob}($ car2 | [showgoat1 \& picks3])

$=$

$[1 \times 1 / 3]$

$\{[0 \times 1 / 3]+[1 \times 1 / 3]+[1 / 2 \times 1 / 3]\}$

$=2 / 3$

The value of the other conditional probability Prob(car3 | [showgoat1 \& picks3]) can be determined by using another instance of the Bayes theorem formula. But it can also be determined from the value of Prob(car3 | [showgoat1 \& picks3]). Since by the Total Probability rule

(3)

$\operatorname{Prob}($ car1 $\mid$ [showgoat1 \& picks3]) +Prob(car2 |[showgoat1 \& picks3]) + Prob(car3 | [showgoat 1 \& picks3]) $=1$

and

(4)

$\operatorname{Prob}(\operatorname{car} 1 \mid$ [showgoat1 \& picks3]) $=0$

- volume 4, no. 1 (Winter 2012) 
from equation (2) we have

(5)

$\operatorname{Prob}($ car3 $\mid$ [showgoat1 \& picks3]) $=1 / 3$

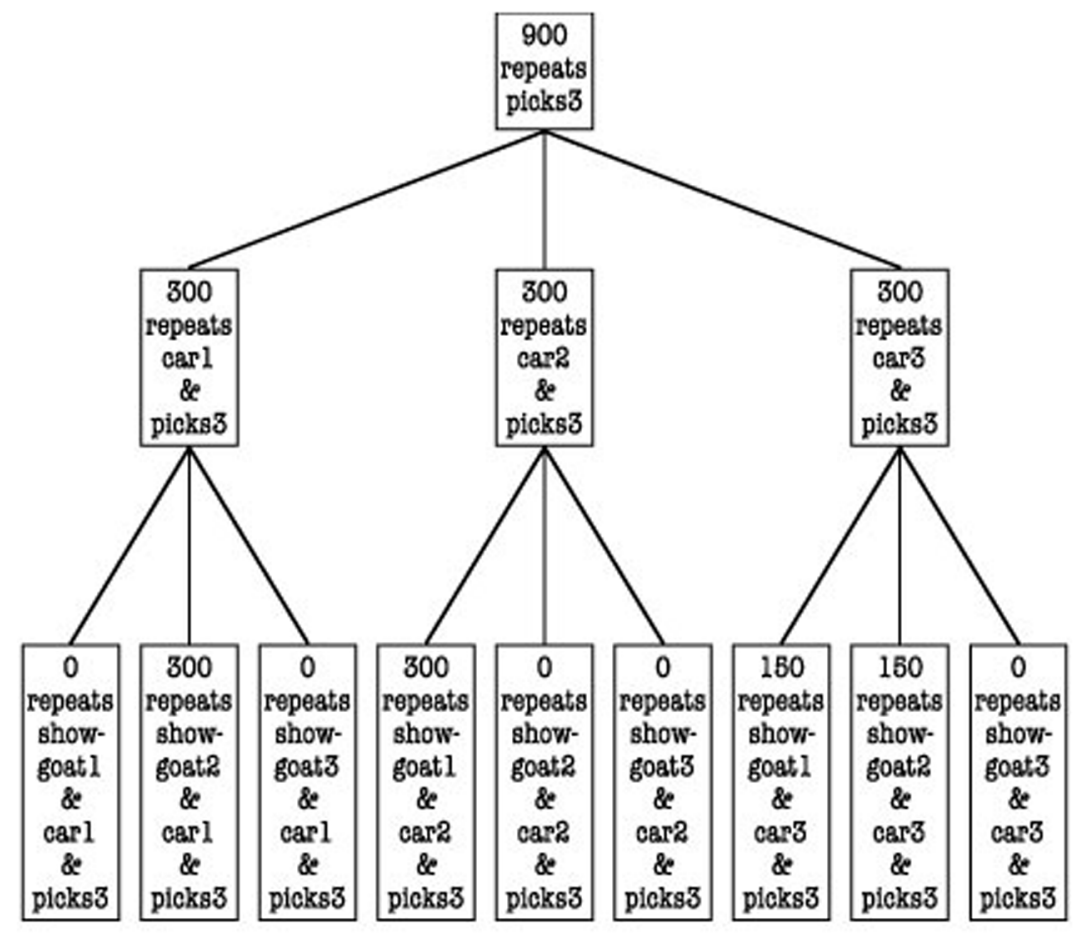

Monty Hall Problem Frequence Tree

Some people are more easily persuaded that these probability values are right when the Bayes theorem reasoning about chances is turned into reasoning about "natural frequencies" (Gigerenzer and Edwards, 2003). For the Monty Hall puzzle this method turns the question "Do you increase your chance of getting the car by changing from your initial choice of door number three to door number two?" into "In many repetitions of this problem would you get a car more often by changing from your initial choice of door number three to door number two?" Suppose, for example, the problem is repeated 900 times. In all of these 900 instances you pick door number three at the first step (see the box at the top of the diagram above, "Monty Hall frequency tree") and in some but not all the host then opens door number one and shows you a goat (see the bottom row of boxes in the diagram). Of the cases where the host opens door number one and shows you a goat, are there more instances in which the car is hidden behind door number two than 
door number three, or are these numbers just the same? Using the abbreviations above, according to this way of putting the Bayes theorem explanation, the answer is given by figuring out that

(6)

$\operatorname{Prob}($ car2 | [showgoat1 \& picks3])

$=$

(Total number of repeats when car2 \& showgoat1 \& picks3)

(Total number of repeats when showgoat1 \& picks3)

$=$

300

$(0+300+150)$

$=2 / 3$

and

(7)

$\operatorname{Prob}($ car3 | [showgoat1 \& picks3])

$=$

(Total number of repeats when car3 \& showgoat1 \& picks3)

(Total number of repeats when showgoat1 \& picks3)

$=$

150

$(0+300+150)$

$=1 / 3$

The frequency-partitioning tree graph makes it easy to calculate the relevant total number of repeats or counts. (Challenge to the reader: Why are the counts in the second and third rows of boxes in the tree as they are? Hint: See the assumptions listed below.)

As Rosenhouse points out, this Bayes theorem justification for the numbers - whether it uses probabilities or turns everything into frequencies—-makes some assumptions which 
are not all obvious or explicit in the classical statement of the problem.

- [Assumption A] After the host has opened a door, the chance the car is behind door number two is equal to or is a disguised way of identifying a conditional posterior probability - the one given in equation (1) - or its equivalent relative frequency - that given in equation (6). A similar assumption is made about the probability or chance it is instead behind door number two.

- [Assumption B] The prior probabilties that the car is behind door number one or number two or number three are all the same, and so each have a value of one-third.

- [Assumption C] Monty Hall-the host-will not open a door and show a car. It's not merely that this does not happen by accident. The rule is that it cannot happen.

- [Assumption D] Monty Hall will never open a door chosen by you-the contestant-at the first stage. In the classic statement you chose door number three at the first stage. This assumption means that Monty Hall will not open number three, even if a goat is behind it.

- [Assumption E] When, consistent with other assumptions, Monty Hall is left with more than one option for a door to open, he always randomizes equally amongst the options. Monty Hall, for example, does not prefer to open even-numbered rather than odd-numbered doors when both types are left available to him as an option.

Assumption $A$ has been argued against by some philosophers. Rosenhouse shows these arguments against $A$ are wrong in his last chapter. Assumptions $B$ through $E$ are the ones used to get the values in equation (2). What changing each of these numbers does to the accepted solution is investigated in various different spots in Rosenhouse's book.

Many writers believe some or all of these particular assumptions are part of the classical statement of the puzzle even though they are not explicitly there. They are hidden or implicit. That would mean changing any of them changes the problem. Rosenhouse, for example, thinks Assumption E isn't hidden or implicit in the classical statement of the problem but that the rest of the assumptions are. I'm not sure that this can be entirely correct. The next paragraph describes another puzzle that sounds a lot like the Monty Hall problem in its classical form. One of the assumptions listed above for the Bayes theorem solution to the Monty Hall problem should not be made in this problem. If that assumption should not be made, then it is not hidden in the statement of this other problem. And then it is hard to see which words in the classic statement of the Monty Hall puzzle make it an implicit hidden part of that problem. I think the same point holds for other assumptions too. This is not to say that the assumptions are unnatural or incorrect. The point is that they are all extra additions to or clarifications of the classical statement of the Monty Hall problem. 
This other puzzle comes from Marilyn vos Savant's popular newspaper magazine column "Ask Marilyn" (May 18, 2003), the same column that started many of the debates about the Monty Hall puzzle. Somehow Rosenhouse missed this one in his otherwise exhaustive investigation of the literature on the Monty Hall problem.

You are taking a multiple choice test with one question. The available answers are labelled "a", "b", and "c". One of the answers is right and the other two are wrong. The test setup is a little unorthodox. There are two stages. You pick one of the answers at the first stage or step. Imagine you choose answer c purely at random. (You choose at random because you have not studied.) After looking at your choice, the instructor or test supervisor says to you and everyone else taking the test that the answer labelled "a" is wrong. Now comes the second stage. You can switch your choice of answers. Answer a has been eliminated but two answers remain. If you first chose answer $c$, for example, you can now switch to the other remaining answer b. Does switching give you a better chance of ending up with the right answer?

(Challenges to the reader: Which assumption about the test supervisor from $A$ through E should not be made in this problem? How would the numbers in a frequency tree for this problem differ from those in the frequency tree for the Monty Hall puzzle? )

The first obvious question about the Monty Hall problem was: What is the correct solution and why is that solution right? There is a second obvious question. Very few people arrive at this solution-that you double your chance of winning the car by switching doors-on their own. The commonly given answer is that the probability of winning the car is the same whether you switch or stay. And even after the correct or accepted solution is provided almost everyone finds it hard to believe. So the second question is: Why do people think this way? Why do even some mathematicians think this way? What are they thinking when they arrive at the common solution and what makes the correct solution so counter-intuitive? This topic too is pretty thoroughly considered in Rosenhouse's survey.

Much of the research done by cognitive scientists and psychologists summarized by Rosenhouse looks at the possibility that all those who think the chance or probability of winning the car is the same whether you switch or stay employ some common shared way of thinking about the problem.

For example, they might be reasoning something like this: What the host does clearly makes no difference to the actual location of the hidden car or the goats; that's all set at the beginning. So what the host does can't make any difference to the chance or probability you have of winning the car by staying with your initially chosen door or switching. In other words

(8)

Since Prob(car3 $\mid$ picks3) = Prob(car2 | picks3) at the start when the contests picks door 
\#3 and nothing happens to alter anything about where the car is, it must be that after the host opens door \#1 and shows a goat Prob(car3 | [showgoat1 \& picks3]) = Prob(car2 | [showgoat1 \& picks3])

Hence, the chance or probability of winning the car is the same whether you switch or stay.

But if that's really the way these people are thinking, then it seems like it should not be affected by changing the number of doors in the Monty Hall problem-for example, from the classical 3 to 30 or even 300 different doors, with the host opening all but two doors and showing that goats are behind each opened one. Whether there are 3 or 300 doors, what the host does makes no change to the physical location of the car or the goats. But the psychological research described by Rosenhouse shows that people will much more often think that you do increase your chance of winning the car by switching doors when the number of doors is increased than when it is left at the classical 3 doors!

Given this kind of data, if there is some common way of thinking about the Monty Hall problem shared by those who think the chance or probability of winning the car is the same whether you switch or stay, it seems like it is not the one just described and it is very hard to figure out exactly what that is.

But there is another challenge for cognitive psychology researchers. Even after hearing the Bayes theorem formula explanation for the accepted or correct solution-whether it uses probabilities or turns everything into frequencies - it is hard for many people to believe you double your chance of winning the car by switching doors. The situation with some other probability puzzles is different. Tversky and Kahneman (1983) famously demonstrated that many people think

(9) Linda is a bank teller

is more probable or likely to be true than

(10) Linda is a bank teller who is active in a feminist movement.

This can't be correct. But once it is explained to people why it can't be correct they change their minds. They realize that (10) has to be less probable than (9). In the Monty Hall puzzle, the idea that the chance or probability of winning the car is the same whether you switch or stay is almost ineradicable.

One way of putting the issue is this: Suppose people do use some rule or way of thinking to arrive at the incorrect conclusion that the chance or probability of winning the car is the same whether you switch or stay. After learning that this rule leads them 
to make a mistake, why can't they give up the rule and accept the results of the Bayes theorem formula explanation? About this part of the second question there also does not yet seem to be an answer in the psychological and cognitive science research ably and enjoyably summarized by Rosenhouse.

\section{References}

Gigerenzer, G., \& Edwards, A. (2003). Simple tools for understanding risks: from unnumeracy to insight. British Medical Journal, 327.

Tversky, A., \& Kahneman, D. (1983). Extensional versus intuitive reasoning:The conjunction fallacy in probability judgment. Psychological Review, 90.

vos Savant, Marilyn (May 18, 2003). Ask Marilyn column. Parade Magazine.

- volume 4, no. 1 (Winter 2012) 